\title{
Medical and Economic Burden of Heparin-Induced Thrombocytopenia: A Retrospective Nationwide Inpatient Sample (NIS) Study
}

\author{
Ranjan Pathak, MD*, Vijaya Raj Bhatt, MBBS², Paras Karmacharya, MD¹, Madan Raj Aryal, MD', Anthony A. Donato, MD, MHPE1 \\ ${ }^{1}$ Department of Internal Medicine, Reading Health System, West Reading, Pennsylvania; ${ }^{2}$ Division of Hematology-Oncology, Department of Internal \\ Medicine, University of Nebraska Medical Center, Omaha, Nebraska.
}

In recent years, US hospitals have switched from use of unfractionated heparin to use of low-molecular-weight heparin, which is associated with lower risk of heparin-induced thrombocytopenia (HIT). In the study reported here, we retrospectively searched the Nationwide Inpatient Sample (NIS) for patients who were at least 18 years old and received a diagnosis of HIT between 2009 and 2011. Our goal was to get an updated perspective on the incidence and economic impact of HIT. We calculated the incidence of HIT overall and in subgroups of patients who underwent cardiac, vascular, or orthopedic surgery. We compared characteristics of patients with and without HIT and compared characteristics of patients with HIT with thrombosis (HITT) and HIT patients without thrombosis. Of 98,636,364 hospitalizations, 72,515 (0.07\%) involved
HIT. Arterial and venous thromboses were identified in 24,880 (34.3\%) of HIT cases. Men were slightly more likely to have a HIT diagnosis (50.1\%), but women had higher rates of HIT after cardiac surgery (odds ratio [OR], 1.41; 95\% confidence interval $[\mathrm{Cl}], 1.26-1.58)$ and vascular surgery (OR, 1.42; 95\% Cl, 1.291.57). Rates of HIT were $0.53 \%$ (95\% Cl, $0.51 \%-0.54 \%)$ after cardiac surgery, $0.28 \%(95 \% \mathrm{Cl}, 0.28 \%-0.29 \%)$ after vascular surgery, and $0.05 \%(95 \% \mathrm{Cl}, 0.05 \%-0.06 \%)$ after orthopedic surgery. HIT and HITT cases were significantly $(P<0.001)$ more likely than non-HIT cases to be fatal $(9.63 \%$ and $12.28 \%$ vs $2.19 \%$ ), and they had significantly higher costs and longer inpatient stays. HIT and especially HITT are associated with increased mortality, costs, and length of stay. Journal of Hospital Medicine 2017;12:94-97. (C) 2017 Society of Hospital Medicine
Each year, approximately one-third of all hospitalized medical and surgical patients in the United States (about 12 million patients) are exposed to heparin products for the prevention or treatment of thromboembolism. ${ }^{1}$ Although generally safe, heparin can trigger an immune response in which platelet factor 4-heparin complexes set off an antibody-mediated cascade that can result in heparin-induced thrombocytopenia (HIT) and paradoxical arterial and venous thromboses, or heparin-induced thrombocytopenia with thrombosis (HITT). The incidence of HIT appears to be significantly higher with the more immunogenic unfractionated heparin (UFH) (2\%-3\% if treated for $\geq 5$ days) than with low-molecular-weight heparin (LMWH) $(0.2 \%-0.6 \%)^{2}$ and is significantly higher in postoperative patients $(1 \%$ $5 \%$ ) than in medical patients. ${ }^{3}$ Older patients and female patients, especially those who undergo surgery, are thought to be at higher risk. ${ }^{4}$ Progression from HIT to HITT can occur in up to $50 \%$ of surgical patients, ${ }^{5}$ and HITT can significantly increase mortality. ${ }^{4}$

In the United States, LMWH use has increased 5-fold since 2000_ an increase attributed to the 2010 release of generic enoxaparin. ${ }^{6}$ As US hospitals switch from UFH to LMWH

\footnotetext{
*Address for correspondence and reprint requests: Ranjan Pathak, MD, Department of Internal Medicine, Reading Health System, 6th Ave \& Spruce St, West Reading, PA; Telephone: 484-628-5455; Fax: 484-628-9003; E-mail: ranjanrp@gmail.com

Additional Supporting Information may be found in the online version of this article.
}

Received: January 6, 2016; Revised: June 9, 2016; Accepted: June 23, 2016 2017 Society of Hospital Medicine DOI 10.12788/jhm.2687 with its significantly lower risk of HIT, up-to-date HIT incidence data may help physicians and payers better understand the impact of the disorder on mortality and hospital length of stay (LOS) for medical patients and subsets of surgical patients and subsequently direct screening efforts to those at highest risk. Therefore, in the present study, we used national data to determine the latest incidence and economic implications of HIT overall and for high-risk surgical groups.

\section{METHODS}

In this study, we analyzed data from the Nationwide Inpatient Sample (NIS) database, part of the Healthcare Cost and Utilization Project (HCUP) of the Agency for Healthcare Research and Quality (AHRQ). The period studied was 2009-2011. We used International Classification of Diseases, Ninth Revision, Clinical Modification (ICD-9-CM) code 289.84, introduced in 2009, to identify patients who were at least 18 years old and had a primary or secondary diagnosis of HIT. Validated Clinical Classifications Software (CCS) was used to identify those who underwent cardiac, vascular, or orthopedic surgery, and ICD-9-CM codes for various thromboses were used to identify those with HITT (Supplemental Figure, Supplemental Table 1). Baseline patient and hospital characteristics were compared using the Pearson's Chi-square test for categorical variables and the Student $t$ test for continuous variables ( 2 -sided $P<0.05$ for statistical significance) (Table 1). We calculated the incidence of HIT overall and for the 3 surgical subgroups and compared the cohorts on their mean hospital LOS, mean hospital charge, and in-hospital mortality (Table 2).

Statistical analysis was performed with Stata Version 13.1 
TABLE 1. Baseline Characteristics of Patients With HIT and HITT

\begin{tabular}{|c|c|c|c|c|c|c|}
\hline Characteristic & $\begin{array}{c}\text { No HIT } \\
(\mathrm{n}=98,563,849)\end{array}$ & $\begin{array}{c}\text { HIT } \\
(\mathrm{n}=72,515)\end{array}$ & $P$ & $\begin{array}{l}\text { HIT w/o thrombosis } \\
\quad(n=47,635)\end{array}$ & $\begin{array}{c}\text { HITT } \\
(n=24,880)\end{array}$ & $P$ \\
\hline Mean (SD) age, y & $57.29(20.79)$ & $65.25(15.64)$ & $<0.001$ & $65.14(15.91)$ & $65.46(15.04)$ & 0.32 \\
\hline Female sex & 59.62 & 49.88 & $<0.001$ & 50.45 & 48.77 & 0.08 \\
\hline Race & & & 0.002 & & & 0.009 \\
\hline White & 68.35 & 67.05 & & 65.76 & 69.56 & \\
\hline Black & 15.03 & 17.12 & & 18.24 & 14.94 & \\
\hline Hispanic & 10.57 & 8.94 & & 9.12 & 8.59 & \\
\hline Other/unknown & 6.05 & 6.89 & & 6.88 & 6.91 & \\
\hline Insurance & & & $<0.001$ & & & $<0.001$ \\
\hline Medicare & 45.22 & 63.02 & & 65.34 & 58.58 & \\
\hline Medicaid & 15.45 & 9.78 & & 9.87 & 9.62 & \\
\hline Private & 29.86 & 21.22 & & 19.57 & 24.56 & \\
\hline Self-pay & 5.57 & 3.09 & & 2.66 & 3.91 & \\
\hline No charge & 0.57 & 0.47 & & 0.42 & 0.57 & \\
\hline Other & 3.33 & 2.41 & & 2.24 & 2.76 & \\
\hline Region & & & 0.15 & & & 0.07 \\
\hline Northeast & 20.05 & 17.79 & & 18.22 & 16.96 & \\
\hline Midwest & 23.08 & 21.31 & & 20.77 & 22.35 & \\
\hline South & 38.35 & 43.10 & & 43.55 & 42.23 & \\
\hline West & 18.52 & 17.81 & & 17.47 & 18.46 & \\
\hline Hospital location/teaching status & & & $<0.001$ & & & 0.21 \\
\hline Rural & 12.46 & 5.10 & & 5.29 & 4.72 & \\
\hline Urban/nonteaching & 41.80 & 38.68 & & 39.01 & 38.05 & \\
\hline Urban/teaching & 45.75 & 56.23 & & 55.70 & 57.23 & \\
\hline Hospital size (number of beds) & & & $<0.001$ & & & $<0.001$ \\
\hline Small (1-49) & 12.36 & 10.44 & & 10.94 & 9.49 & \\
\hline Medium (50-99) & 23.87 & 20.29 & & 21.12 & 18.70 & \\
\hline Large (100+) & 63.77 & 69.26 & & 67.93 & 71.81 & \\
\hline
\end{tabular}

NOTE: Abbreviations: HIT, heparin-induced thrombocytopenia; HITT, heparin-induced thrombocytopenia with thrombosis.

(Stata Corp, College Station, TX). Survey commands were used to account for the complex survey design in NIS. Reading Health System's Institutional Review Board determined that our study protocol was exempt.

\section{RESULTS}

Of 98,636,364 hospitalizations, $72,515(0.07 \%)$ involved HIT. There were no significant differences in the annual incidence of HIT during the study period $(0.06 \%$ in 2009 , $0.05 \%$ in $2010,0.06 \%$ in 2011).

Patients with HIT were older than patients without HIT (mean age, 65.3 vs 57.3 years; $P<0.001$ ). HIT was slightly more common in men overall (OR, 1.48; 95\% CI, 1.46-1.51), but subgroup analyses revealed women had higher rates of HIT after cardiac surgery (OR, 1.41; 95\% CI, 1.26-1.58) and vascular surgery (OR, 1.42; 95\% CI, 1.29-1.57), though not after orthopedic surgery (OR, 1.06; 95\% CI, 0.89-1.26). The majority of HIT cases were in urban teaching hospitals $(56.23 \%)$ and in large hospitals, those with at least 325 beds $(69.26 \%)$. There was no difference in mean age between patients with HITT and patients with HIT without thrombosis (65.46 vs 65.14 years; $P=0.32$ ). Although the incidence of HITT did not differ by hospital location or teaching status, HITT cases were more common in hospitals with at least 325 beds (71.81\%).
Regarding HIT, the death rate was 4-fold higher for patients with the disorder $(9.63 \%)$ than for those without it (2.19\%); hospital LOS and costs were significantly higher, too (Table 2). In addition, in-hospital mortality was higher $(P<0.001)$ for patients with HITT $(12.28 \%)$ than for patients with HIT without thrombosis (8.24\%); HITT patients' hospital LOS and costs were higher as well. In patients who had cardiac, vascular, or orthopedic surgery, development of HIT was also associated with significantly higher in-hospital mortality, mean hospital LOS, and mean hospital charge. In patients with HITT, deep vein thrombosis (DVT) and pulmonary embolism represented the majority of reported cases (Supplemental Table 2). However, in patients who had cardiac surgery, acute arterial thromboses of coronary and cerebral vessels were more common.

\section{DISCUSSION}

In this national database survey, the overall incidence of HIT during the study period $2009-2011$ was $0.07 \%$, or 1 in 1350 hospitalized patients. Although earlier studies reported rates as high as $5 \%$ for high-risk subgroups of surgical patients, ${ }^{7}$ our data are more in line with more recently reported rates: about $0.02 \%$ for hospital admissions ${ }^{8}$ and from less than $0.1 \%$ to $0.4 \%$ for patients who received heparin. ${ }^{9}$ 
TABLE 2. In-Hospital Mortality, Mean Hospital LOS, and Mean Hospital Charge for Patients With HIT and HITT, Overall and in Cardiac, Vascular, and Orthopedic Surgery

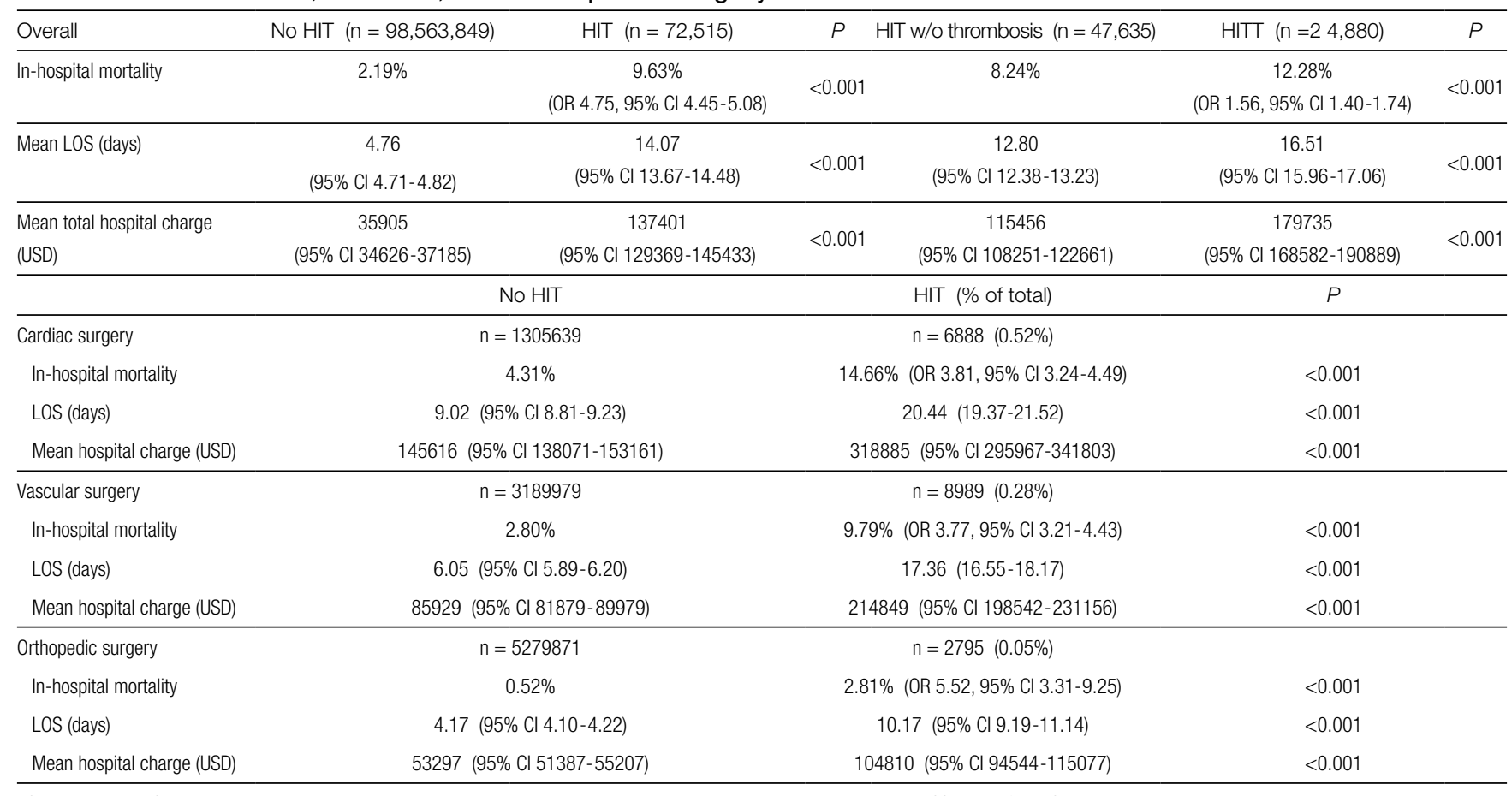

NOTE: Abbreviations: Cl, confidence interval; HIT, heparin-induced thrombocytopenia; HITT, heparin-induced thrombocytopenia with thrombosis; LOS, length of stay; OR, odds ratio.

Older studies, which predominantly involved postoperative patients and were conducted when UFH often was the first-line heparin product used, may account for higher rates relative to ours. Of the 3 types of surgeries we evaluated, cardiac surgery had the highest HIT rate $(0.5 \%)$, consistent with other studies. ${ }^{4}$ The higher HIT/HITT rates found for larger urban hospitals in our study might be attributable to increased awareness and testing, availability of hematology consultation, and higher risk of heparin use in this setting, where patients are sicker and cases and procedures more complicated.

Age was an important determinant of HIT risk in our study and in similar large-database series. ${ }^{4}$ Whether increased UFH use in the elderly (because of age or kidney disease) was a causative factor in this finding is unknown. In our study, although men and women had a nearly equal incidence of HIT, women had a significantly higher risk of HIT after both cardiac surgery and vascular surgery. Immune-mediated mechanisms that are more common in females may play a causative role in these settings. ${ }^{10}$

Our study results showed HIT associated with increased hospital LOS and an almost 4-fold increase in inpatient mortality and costs. The increased economic burden in HIT cases may be driven by the diagnostic work-up cost and expensive alternative anticoagulation. ${ }^{11,12}$ Similarly, compared with HIT without thrombosis, HITT was associated with significantly increased hospital LOS (3.7 days), total hospital charge $(\$ 64,279)$ and mortality $(49 \%$ increase, to $12.2 \%$ from $8.2 \%$ ), consistent with prior studies. ${ }^{13}$ In addi- tion, $34.1 \%(24,704)$ of our HIT patients developed at least 1 thrombotic complication, with venous thromboses more common than arterial thromboses, as previously reported..$^{13}$ Lower extremity DVT was the most common thrombosis in orthopedic and vascular surgery. However, in cardiac surgery, acute coronary occlusion was the most common thrombotic complication. We postulate that the difference stems from the increased propensity of HIT-related thrombosis to occur in areas of vascular injury. ${ }^{14}$

The strengths of our study include its large size, which increases the generalizability of its results and avoids the biases inherent in small, single-center studies. As with any administrative dataset, the NIS may include coding errors related to underdiagnosis and overdiagnosis (eg, a HIT/ HITT diagnosis carried forward from prior episodes). In our study, we inferred the HITT diagnosis in HIT cases with a vascular complication, but we could have missed HIT cases that had not been coded for vascular complications, and we could have overassociated vascular complications that had predated HIT and been treated with heparin. Although HIT and HITT were associated with worse clinical outcomes and increased hospital LOS, it is possible patients who were hospitalized longer had more opportunities for heparin use, and this exposure led to HIT or HITT. The lack of details regarding prior heparin use, including type of heparin (UFH or LMWH), prevented us from inferring the actual risks of individual heparin products.

In conclusion, in cardiac, vascular, and orthopedic surgery, HIT and especially HITT can significantly increase hospital 
LOS, inpatient costs, and mortality. Lower extremity DVT and acute coronary artery occlusion are the most common thrombotic complications in these cases. HIT screening strategies that incorporate platelet counts are recommended only in patients at highest risk $(>1 \%)$, according to the most recent American College of Chest Physicians guidelines, but this recommendation was made on the basis of the high cost of alternative anticoagulants. Given our more recent data regarding the very high costs of HIT and especially HITT, screening strategies with platelet counts may prove more cost-effective. Recent genome-wide studies that found higher rates of HIT in patients with T-cell death-associated gene 8 (TDAG8) may help explain sex differences in postoperative patients and identify patients at highest risk so alternative anticoagulants can be used. ${ }^{15}$

Disclosures: This study was funded by the Reading Health System (grant RHS0010). Dr. Bhatt is supported by the Physician-Scientist Training Program (grant 2015 2016), College of Medicine, University of Nebraska Medical Center. The other authors report no financial conflicts of interest.

\section{References}

1. Fahey VA. Heparin-induced thrombocytopenia. J Vasc Nurs. 1995;13(4):112-116

2. TE, Greinacher A. Heparin-induced thrombocytopenia and cardiac surgery. Ann Thorac Surg. 2003;76(6):2121-2131.

3. Junqueira DR, Perini E, Penholati RR, Carvalho MG. Unfractionated heparin versus low molecular weight heparin for avoiding heparin-induced thrombocytopenia in postoperative patients. Cochrane Database Syst Rev. 2012;(9):CD007557.

4. Seigerman M, Cavallaro P, Itagaki S, Chung I, Chikwe J. Incidence and outcomes of heparin-induced thrombocytopenia in patients undergoing cardiac surgery in
North America: an analysis of the Nationwide Inpatient Sample. J Cardiothorac Vasc Anesth. 2014;28(1):98-102.

5. Greinacher A. Heparin-induced thrombocytopenia. N Engl J Med. 2015;373(3): 252-261.

6. Grabowski HG, Guha R, Salgado M. Regulatory and cost barriers are likely to limit biosimilar development and expected savings in the near future. Health Aff (Millwood). 2014;33(6):1048-1057.

7. Prandoni P, Siragusa S, Girolami B, Fabris F; BELZONI Investigators Group. The incidence of heparin-induced thrombocytopenia in medical patients treated with low-molecular-weight heparin: a prospective cohort study. Blood. 2005;106(9):3049-3054.

8. Jenkins I, Helmons PJ, Martin-Armstrong LM, Montazeri ME, Renvall M. High rates of venous thromboembolism prophylaxis did not increase the incidence of heparin-induced thrombocytopenia. Jt Comm J Qual Patient Saf. 2011;37(4): 163-169.

9. Zhou A, Winkler A, Emamifar A, et al. Is the incidence of heparin-induced thrombocytopenia affected by the increased use of heparin for VTE prophylaxis? Chest. 2012;142(5):1175-1178.

10. Warkentin TE, Sheppard JA, Sigouin CS, Kohlmann T, Eichler P, Greinacher A. Gender imbalance and risk factor interactions in heparin-induced thrombocytopenia. Blood. 2006;108(9):2937-2941.

11. Baroletti S, Piovella C, Fanikos J, Labreche M, Lin J, Goldhaber SZ. Heparin-induced thrombocytopenia (HIT): clinical and economic outcomes. Thromb Haemost. 2008;100(6):1130-1135.

12. Smythe MA, Koerber JM, Fitzgerald M, Mattson JC. The financial impact of heparin-induced thrombocytopenia. Chest. 2008;134(3):568-573.

13. Nand S, Wong W, Yuen B, Yetter A, Schmulbach E, Gross Fisher S. Heparin-induced thrombocytopenia with thrombosis: Incidence, analysis of risk factors, and clinical outcomes in 108 consecutive patients treated at a single institution. Am J Hematol. 1997;56(1):12-16.

14. Hong AP, Cook DJ, Sigouin CS, Warkentin TE. Central venous catheters and upper-extremity deep-vein thrombosis complicating immune heparin-induced thrombocytopenia. Blood. 2003;101(8):3049-3051.

15. Karnes JH, Cronin RM, Rollin J, et al. A genome-wide association study of heparin-induced thrombocytopenia using an electronic medical record. Thromb Haemost. 2015;113(4):772-781. 